Article

\title{
Prognostic Value of Incomplete Revascularization after Percutaneous Coronary Intervention Following Acute Coronary Syndrome: Focus on CKD Patients
}

\author{
Thomas Cardi ${ }^{1}$, Anas Kayali ${ }^{1}$, Antonin Trimaille ${ }^{1, *}$, Benjamin Marchandot ${ }^{1}$, Jessica Ristorto ${ }^{1}$, \\ Viet Anh Hoang ${ }^{1,2}$, Sébastien Hess ${ }^{1}$, Marion Kibler ${ }^{1}$, Laurence Jesel ${ }^{1,3}$, Patrick Ohlmann ${ }^{1}$ and \\ Olivier Morel ${ }^{1,3}$ \\ 1 Pôle d'Activité Médico-Chirurgicale Cardio-Vasculaire, Nouvel Hôpital Civil, Centre Hospitalier \\ Universitaire, Université de Strasbourg, 67090 Strasbourg, France; thomas.cardi@chru-strasbourg.fr (T.C.); \\ anas.kayali@chru-strasbourg.fr (A.K.); benjamin.marchandot@chru-strasbourg.fr (B.M.); \\ jessica.ristorto@chru-strasbourg.fr (J.R.); vietanhhoang78@yahoo.fr (V.A.H.); \\ sebastien.hess@chru-strasbourg.fr (S.H.); mario.kibler@chru-strasbourg.fr (M.K.); \\ laurence.jesel@chru-strasbourg.fr (L.J.); patrick.ohlmann@chru-strasbourg.fr (P.O.); \\ olivier.morel@chru-strasbourg.fr (O.M.) \\ 2 Vietnam National Heart Institute, Bach Mai Hospital, 78 Giai Phong, Dong Da, 10000 Hanoi, Vietnam \\ 3 Regenerative Nanomedicine, UMR 1260, INSERM (French National Institute of Health and Medical \\ Research), FMTS (Fédération de Médecine Translationnelle de l'Université de Strasbourg), \\ Université de Strasbourg, Faculté de Médecine, 11 rue Humann, 67085 Strasbourg, France \\ * Correspondence: antonin.trimaille@chru-strasbourg.fr; Tel.: +00-33-369-551-055; Fax: +00-33-369-551-736
}

Received: 22 April 2019; Accepted: 3 June 2019; Published: 6 June 2019

Abstract: Background: Residual coronary artery disease (CAD) has been associated with worsened prognosis in patients undergoing percutaneous coronary intervention (PCI) for acute coronary syndromes (ACS). The residual SYNTAX Score (rSS) aims to assess residual CAD after PCI. The association between kidney function and rSS has not been investigated in ACS patients. In this study, we sought to determine whether chronic kidney disease (CKD) patients exhibit more incomplete revascularization following stage revascularization procedures by PCI. We evaluated the impact of incomplete revascularization on the occurrence of major cardiovascular events (MACE) at one-year follow-up. Methods: A total of 831 ACS patients undergoing PCI were divided into 3 subgroups according to their estimated Glomerular Filtration Rate (eGFR): 695 with eGFR $\geq 60 \mathrm{~mL} / \mathrm{min} / 1.73 \mathrm{~m}^{2}$, 108 with eGFR $60-30 \mathrm{~mL} / \mathrm{min} / 1.73 \mathrm{~m}^{2}, 28$ with eGFR $<30 \mathrm{~mL} / \mathrm{min} / 1.73 \mathrm{~m}^{2}$. Initial SYNTAX score (SS) and rSS were calculated for all patients. Incomplete revascularization was defined by rSS $>8$. The primary endpoint was the occurrence of MACE (all-cause mortality, myocardial infarction (MI), repeated revascularization except from planned revascularization, stroke and definite or probable recurrent stent thrombosis) one year after the index procedure. Results: Severe CKD patients had significantly higher MACE $(12.0 \%$ vs. $25.9 \%$ vs. $35.7 \% ; p<0.001)$, all-cause mortality, cardiovascular mortality and heart failure events. Patients with $\mathrm{rSS}>8$ had higher MACE, all-cause and cardiovascular mortality. CKD was an independent predictive factor of rSS > 8 (HR: 1.65, 95\% CI: 1.01 to 2.71; $p=0.048$ ). Multivariate analysis identified $\mathrm{rSS}>8$, but not CKD, as an independent predictor of cardiac death and MACE. Conclusion: In ACS, CKD is predictive of incomplete revascularization, which stands out as a strong predictor of adverse cardiovascular outcomes including cardiac death and MACE.

Keywords: chronic kidney disease; SYNTAX score; residual SYNTAX score; incomplete revascularization; coronary artery disease 


\section{Introduction}

With respect to the importance of non-culprit lesions in acute coronary syndromes (ACS), the current characterization of residual atherosclerotic burden by the generic denomination "multiple vessel disease" appears insufficient to accurately assess the risk of adverse cardiovascular outcomes [1-3]. Focused attention on concomitant and additional coronary lesions is crucial to guide clinical decisions in the field of coronary artery disease (CAD) and improve mid- and long-term prognostics following percutaneous coronary intervention (PCI). Therefore, the residual SYNTAX Score (rSS) has emerged as an angiographic scoring system aimed to assess residual (CAD) after (PCI) [4]. A recent study has established that a staged PCI that achieves reasonably complete revascularization ( $\mathrm{rSS} \leq 8$ ) improves mid-term survival and reduces the incidence of repeated PCI in patients with ST-segment elevation myocardial infarctions (STEMI) and multivessel disease [5]. By contrast, significant residual CAD assessed by higher rSS, confers a worsened prognosis in patients undergoing PCI. Diabetes, previous myocardial infarction (MI) and multivessel coronary artery disease are currently established as independent predictors of high rSS [1,4]. Up to now, the association between kidney function and rSS has not been adequately investigated in ACS patients. In the present prospective study, we sought to determine the incidence of incomplete revascularization by calculating rSS among chronic kidney disease (CKD) patients with staged revascularization procedures by PCI in ACS. Moreover, we sought to investigate the impact of incomplete revascularization on the occurrence of major adverse cardiovascular events (MACE) at one-year follow-up.

\section{Methods}

\subsection{Patients}

This study enrolled all consecutive ACS patients undergoing PCI at our institution (Nouvel Hôpital Civil, Université de Strasbourg, Strasbourg, France) between February 2014 and February 2016. The study was performed in accordance with the Declaration of Helsinki. The protocol was approved by the ethics committee, and informed written consent was obtained from all patients. The flow-chart of the study is shown in Figure 1.

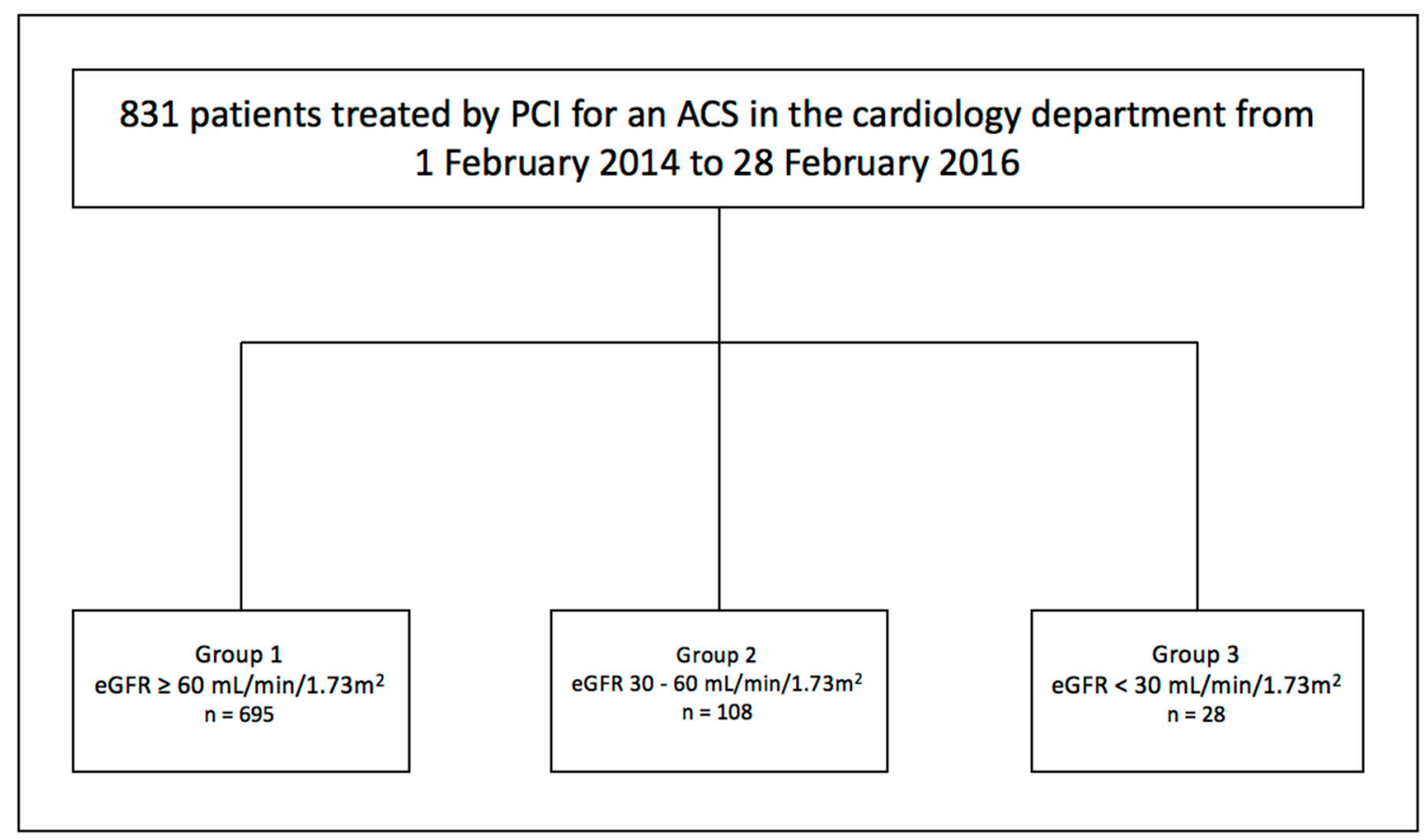

Figure 1. Flow-chart. n: number, ACS: Acute coronary syndrome, eGFR: estimated Glomerular filtration rate, PCI: Percutaneous coronary interventions. 


\subsection{Chronic Kidney Disease Staging}

Baseline serum creatinine levels were assessed at admission in all patients. The estimated glomerular filtration rate (eGFR) was calculated using the MDRD (Modification of Diet in Renal Disease) formula [6]. Patients were divided into 3 subgroups according to their eGFR levels: $\geq 60 \mathrm{~mL} / \mathrm{min} / 1.73 \mathrm{~m}^{2}$; $\geq 30$ and $<60 \mathrm{~mL} / \mathrm{min} / 1.73 \mathrm{~m}^{2}$; and $<30 \mathrm{~mL} / \mathrm{min} / 1.73 \mathrm{~m}^{2}$. Patient's baseline characteristics included age, gender, body mass index (BMI), history of dyslipidemia, smoking status at admission, history of hypertension (HT), history of diabetes mellitus (DM), personal and family history of cardiovascular disease, left ventricle ejection fraction (LVEF) and discharge medication. Patients with prior coronary artery bypass grafting (CABG) were excluded from our analysis.

\subsection{Collection of Data}

Follow-up information was obtained using a written questionnaire via a telephone interview with the cardiologist, referring physician or patient. In the absence of response, the patient's electronic medical file was consulted.

\subsection{Calculation of SYNTAX Score (SS), Residual SYNTAX Score (rSS) and SYNTAX Index Revascularization (SRI)}

The SYNTAX Score (SS) was calculated from the pre-procedural angiogram, in which each coronary lesion producing $>50 \%$ diameter stenosis in vessels $>1.5 \mathrm{~mm}$ by visual estimation was scored separately using the SS algorithm, and added to obtain the overall SS. Staged PCI was performed in patients with angiographic stenosis $\geq 70 \%$ or who demonstrated residual ischemia assessed either by fractional flow reserve (FFR) or by perfusion myocardial tomography. Staged PCI was performed within 30 days following the index ACS. The residual SYNTAX scores (rSS) was defined as the SS recalculated after staged PCI and was calculated in all patients enrolled in this study. To calculate the rSS, the final post-PCI angiogram was scored to assess untreated disease after staged PCI. A dedicated interventional cardiologist, who was blinded to both baseline characteristics and clinical outcomes reviewed all post-procedural angiograms. Similarly, the SYNTAX revascularization index (SRI) which stands as an angiographic index tool aimed to quantify the proportion of revascularized myocardium was calculated and defined as: 100 (1-rSS/baseline SS) (\%).

\subsection{Study Endpoints}

The primary endpoint was the major adverse cardiac events (MACE) rate, which was defined as the composite of all-cause mortality, myocardial infarction (MI), repeat revascularization except from planned revascularization, stroke and probable or definite recurrent stent thrombosis at one-year follow-up.

The secondary end points were all-cause mortality, cardiovascular mortality (defined as any death with demonstrable cardiovascular cause or any death that was not clearly attributable to a non-cardiovascular cause), ST-segment elevation myocardial infarction (STEMI) defined as a new ST-segment elevation in two consecutive leads associated with increased biochemical myocardial necrosis markers, non-ST-segment elevation myocardial infarction (NSTEMI) defined as the occurrence of ischemic symptoms, ST-segment depression and/or T-wave abnormalities and an increase of biochemical myocardial necrosis markers, stent thrombosis (according to the definition of the Academic Research Consortium), angina, hemorrhagic events (according to Bleeding Academic Research Consortium definition), and rehospitalization for heart failure.

The extent of P2Y12 inhibition by thienopyridines or ticagrelor was established using the vasodilator stimulated phosphoprotein (VASP) assay as previously described $[7,8]$. 


\subsection{Statistical Analysis}

Continuous variables are expressed as mean $+/-$ standard deviation (SD); categorical variables are expressed as frequencies and percentages (\%). Continuous variables between 2 groups were compared by the Student $t$ test or by the Mann-Whitney test as appropriate. Fisher's exact test was used for comparison of categorical variables. Continuous variables were analyzed for normal distribution using the Shapiro-Wilk test. Time to event was defined as the time from PCI to the date of event, with patients censored at death, loss to follow-up, or end of the study. Correlations were calculated using the Spearman test.

Kaplan-Meier analyses were used to construct survival plots of time to death after PCI and compared using the log-rank test.

Multivariate analysis of survival rates was done using Cox models. Variables with $p<0.05$ in univariate analysis were entered into a stepwise ascending multivariate analysis. In addition, variables linked to residual SS were not taken into account in the multivariate analysis for MACE and cardiac death (i.e., three-vessel disease and SS).

The results of the Cox regression are presented as hazard ratios (HR), their 95\% confidence intervals (CIs), and $\mathrm{p}$ values. A $p$ value $<0.05$ was considered statistically significant. Statistical analysis was performed using SPSS version 13.0 software (SPSS Inc., Chicago, IL, USA).

\section{Results}

\subsection{Patient's Baseline Characteristics}

A total of 831 consecutive ACS patients treated with PCI were enrolled in this study. Patients were divided into 3 groups according to their baseline eGFR level: 695 patients with GFR $\geq 60 \mathrm{~mL} / \mathrm{min} / 1.73 \mathrm{~m}^{2}$ were referred to as the "no CKD" group (group 1), 108 patients with GFR $59-30 \mathrm{~mL} / \mathrm{min} / 1.73 \mathrm{~m}^{2}$ as the "moderate CKD" group (group 2) and finally, 28 patients with GFR $<30 \mathrm{~mL} / \mathrm{min} / 1.73 \mathrm{~m}^{2}$ as the "severe $\mathrm{CKD}^{\prime}$ group (group 3). Baseline demographic, clinical, biological and angiographic characteristics are described in Tables 1-3. As previously published by several groups including ours, the extent of P2Y12 inhibition as assessed by platelet reactivity index (PRI) VASP was inversely related to renal function [8].

Table 1. Baseline demographic and clinical characteristics according to renal function.

\begin{tabular}{ccccc}
\hline Variables & eGFR $\geq \mathbf{6 0}$ & eGFR 30-59 & eGFR $<30$ & $p$ Value \\
& $\boldsymbol{n}=\mathbf{6 9 5}$ & $\boldsymbol{n}=\mathbf{1 0 8}$ & $\boldsymbol{n}=\mathbf{2 8}$ & \\
\hline Age & $61.88( \pm 13.15)$ & $76.79( \pm 12.49)$ & $76.43( \pm 12.55)$ & $<0.001$ \\
Gender (female) & $153(22 \%)$ & $38(35.2 \%)$ & $10(35.7 \%)$ & 0.004 \\
STEMI & $392(56.4 \%)$ & $50(46.3 \%)$ & $15(53.6 \%)$ & 0.161 \\
NSTEMI & $268(38.6 \%)$ & $52(48.1 \%)$ & $12(42.9 \%)$ & 0.159 \\
Unstable angina & $35(5 \%)$ & $6(5.6 \%)$ & $1(3.6 \%)$ & 0.911 \\
Diabetes mellitus & $144(20.7 \%)$ & $44(40.7 \%)$ & $12(42.9 \%)$ & $<0.001$ \\
Hypertension & $382(55 \%)$ & $89(82.4 \%)$ & $23(82.1 \%)$ & $<0.001$ \\
Current smoking & $299(43 \%)$ & $19(17.6 \%)$ & $4(14.3 \%)$ & $<0.001$ \\
Dyslipidemia & $314(45.2 \%)$ & $66(61.1 \%)$ & $20(71.4 \%)$ & $<0.001$ \\
Family history of CAD & $160(23 \%)$ & $8(7.4 \%)$ & $4(14.3 \%)$ & 0.001 \\
Prior STEMI & $68(9.80 \%)$ & $14(13 \%)$ & $7(25 \%)$ & 0.028 \\
Prior NSTEMI & $44(6.3 \%)$ & $17(15.7 \%)$ & $6(21.4 \%)$ & $<0.001$ \\
Prior angioplasty & $102(14.7 \%)$ & $24(22.2 \%)$ & $10(35.7 \%)$ & 0.003 \\
Prior Stroke & $35(5 \%)$ & $12(11.1 \%)$ & $6(21.4 \%)$ & $<0.001$ \\
Atrial fibrillation & $40(5.8 \%)$ & $17(15.9 \%)$ & $7(25 \%)$ & $<0.001$ \\
PAD & $41(5.90 \%)$ & $17(15.9 \%)$ & $6(21.4 \%)$ & $<0.001$ \\
Obesity & $188(27.1 \%)$ & $19(17.6 \%)$ & $5(17.9 \%)$ & 0.237 \\
Killip $\geq 2$ & $105(15.1 \%)$ & $44(40.7 \%)$ & $14(50 \%)$ & $<0.001$ \\
LVEF $(\%)$ & $52.51( \pm 11.44)$ & $49.22( \pm 12.87)$ & $45.25( \pm 12.21)$ & $<0.001$ \\
LVEF $\leq 40 \%$ & $125(18 \%)$ & $32(29.6 \%)$ & $12(42.9 \%)$ & $<0.001$ \\
RV dysfunction & $42(6 \%)$ & $11(10.2 \%)$ & $9(32.1 \%)$ & $<0.001$ \\
\hline
\end{tabular}


Table 1. Cont.

\begin{tabular}{ccccc}
\hline Variables & eGFR $\geq \mathbf{6 0}$ & eGFR 30-59 & eGFR $<30$ & $p$ Value \\
& $\boldsymbol{n}=\mathbf{6 9 5}$ & $\boldsymbol{n}=\mathbf{1 0 8}$ & $\boldsymbol{n}=\mathbf{2 8}$ & 0.058 \\
Aspirin & $688(99 \%)$ & $104(96.3 \%)$ & $27(96.4 \%)$ & $<0.001$ \\
Clopidogrel & $248(35.7 \%)$ & $74(68.5 \%)$ & $25(89.3 \%)$ & $<0.001$ \\
Ticagrelor & $188(27.1 \%)$ & $18(16.7 \%)$ & $1(3.6 \%)$ & $<0.001$ \\
Prasugrel & $249(35.80 \%)$ & $12(11.1 \%)$ & $0(0 \%)$ & $<0.001$ \\
ACE-I & $571(82.2 \%)$ & $67(62 \%)$ & $14(50 \%)$ & $<0.001$ \\
ARB & $57(8.2 \%)$ & $20(18.5 \%)$ & $4(14.3 \%)$ & 0.002 \\
Statin & $671(96.5 \%)$ & $97(89.8 \%)$ & $24(85.7 \%)$ & 0.001 \\
Betablocker & $645(92.9 \%)$ & $90(83.3 \%)$ & $23(82.1 \%)$ & 0.003 \\
VKA & $67(9.6 \%)$ & $20(18.5 \%)$ & $9(32.1 \%)$ & 0.007 \\
In hospital hemorrhage & $17(2.4 \%)$ & $7(6.5 \%)$ & $3(10.7 \%)$ & $<0.001$ \\
Acute kidney injury & $54(7.8 \%)$ & $41(38 \%)$ & $14(50 \%)$ & \\
\hline
\end{tabular}

Data are presented as mean \pm SD or $n(\%)$. eGFR is expressed in $\mathrm{mL} / \mathrm{min} / 1.73 \mathrm{~m}^{2}$. eGFR: estimated Glomerular Filtration rate, STEMI: ST elevation myocardial infarction, NSTEMI: non STEMI, PAD: peripheral artery disease, LVEF: Left ventricle ejection fraction, ACE-I: Angiotensin converting enzyme - inhibitors, ARB: Angiotensin II receptor blockers, RV: Right Ventricular, VKA: Vitamin K antagonists.

Table 2. Biological parameters according to renal function.

\begin{tabular}{|c|c|c|c|c|}
\hline Variables & $\begin{array}{c}\text { eGFR } \geq 60 \\
n=695\end{array}$ & $\begin{array}{c}\text { eGFR } 30-59 \\
n=108\end{array}$ & $\begin{array}{c}\text { eGFR }<30 \\
n=28\end{array}$ & $p$ Value \\
\hline Creatinine $(\mu \mathrm{mol} / \mathrm{L})$ & $71.31 \pm 14.82$ & $123.61 \pm 25.76$ & $262.29 \pm 169.98$ & $<0.001$ \\
\hline eGFR $\left(\mathrm{mL} / \mathrm{min} / 1.73 \mathrm{~m}^{2}\right)$ & $85.77 \pm 7.75$ & $48.48 \pm 8.13$ & $21.93 \pm 8.44$ & $<0.001$ \\
\hline Troponin at admission $(\mu \mathrm{g} / \mathrm{L})$ & $10.01 \pm 34.72$ & $13.75 \pm 36.54$ & $15.56 \pm 26.44$ & 0.436 \\
\hline Troponin peak $(\mu \mathrm{g} / \mathrm{L})$ & $70.93 \pm 130.26$ & $67.14 \pm 123.98$ & $75.24 \pm 121.28$ & 0.942 \\
\hline $\mathrm{BNP}(\mu \mathrm{g} / \mathrm{L})$ & $139.87 \pm 253.3$ & $462.74 \pm 640.46$ & $639.07 \pm 599.53$ & $<0.001$ \\
\hline CRP (mg/L) & $11.69 \pm 27.21$ & $34.72 \pm 57.78$ & $72.44 \pm 92.99$ & $<0.001$ \\
\hline Total-cholesterol (mg/dL) & $1.76 \pm 0.58$ & $1.56 \pm 0.6$ & $1.35 \pm 0.69$ & $<0.001$ \\
\hline LDL-C $(\mathrm{mg} / \mathrm{dL})$ & $1.09 \pm 0.44$ & $0.92 \pm 0.45$ & $0.76 \pm 0.45$ & $<0.001$ \\
\hline HDL-C (mg/dL) & $0.39 \pm 0.15$ & $0.4 \pm 0.17$ & $0.33 \pm 0.16$ & 0.065 \\
\hline Triglycerid (mg/dL) & $1.37 \pm 0.9$ & $1.14 \pm 0.66$ & $1.14 \pm 0.93$ & 0.019 \\
\hline Glycated Hemoglobin (\%) & $5.55 \pm 1.9$ & $5.58 \pm 2.16$ & $5.57 \pm 2.09$ & 0.994 \\
\hline Hemoglobin $(\mathrm{g} / \mathrm{dL})$ & $14.23 \pm 1.69$ & $13.15 \pm 2.07$ & $11.32 \pm 2.16$ & $<0.001$ \\
\hline White blood cell count $\left(\times 10^{9} / \mathrm{L}\right)$ & $10.8 \pm 4.07$ & $11.22 \pm 5.87$ & $11.92 \pm 5.98$ & 0.323 \\
\hline Platelets $\left(\times 10^{9} / \mathrm{L}\right)$ & $242.5 \pm 67.17$ & $225.36 \pm 90.02$ & $222.64 \pm 72.34$ & 0.028 \\
\hline VASP (\%) & $23.61 \pm 25.53$ & $28.47 \pm 29.19$ & $48.44 \pm 33.04$ & $<0.001$ \\
\hline
\end{tabular}

Data are expressed as mean \pm SD. eGFR is expressed in $\mathrm{mL} / \mathrm{min} / 1.73 \mathrm{~m}^{2}$. eGFR: estimated Glomerular Filtration rate, BNP: Brain natriuretic peptide, CRP: C-reactive protein, LDL-C: Low-Density Lipoprotein Cholesterol, HDL-C: High-Density Lipoprotein-Cholesterol, VASP: vasodilator stimulated phosphoprotein.

Compared to the "no CKD" group, patients with advanced CKD were older, showed increased prevalence of cardiovascular risk factors including diabetes mellitus, hypertension, dyslipidemia and displayed lower LVEF and more frequent right ventricular dysfunction. As expected, a history of cardiovascular disease was more frequently recorded in CKD patients. Likewise, higher burden of CAD could be demonstrated at baseline in CKD patients with higher SS at baseline and increased incidence of three-vessel coronary artery disease, as reported previously $[9,10]$. Staged procedures were achieved similarly in $24.9 \%$ of patients with GFR $\geq 60 \mathrm{~mL} / \mathrm{min} / 1.73 \mathrm{~m}^{2}, 29 \%$ of patients with GFR $30-59 \mathrm{~mL} / \mathrm{min} / 1.73 \mathrm{~m}^{2}$ and $21.4 \%$ of patients with GFR $<30 \mathrm{~mL} / \mathrm{min} / 1.73 \mathrm{~m}^{2}(p=0.593)$. As expected, a significant relationship between baseline SS and rSS could be established $(r=0.535 ; p<0.001)$. The mean SS was $13 \pm 8$ in patients with one- or two-vessel disease and $25 \pm 10$ in three-vessel disease $(p<0.001)$. Likewise, rSS was $2.9 \pm 4.8$ in patients with one or two-vessel disease and $8.6 \pm 8.3$ in patients with three-vessel disease $(p<0.001)$. Following staged procedures, higher rSS and lower SRI could be evidenced as eGFR declined (Figure 2). The proportions of rSS $>8$ among the 3 groups were, respectively, $16.3 \%, 33.3 \%$ and $46.4 \%$ ( $p<0.001)$. A 2 - to 3-fold elevation of incomplete revascularization 
rate was evidenced as kidney function declined (Table 3). eGFR was inversely related to baseline SS $(r=-0.163 ; p<0.001)$ and $\mathrm{rSS}(r=-0.172 ; p<0.001)$.

Table 3. Angiographic and PCI procedure characteristics according to renal function.

\begin{tabular}{|c|c|c|c|c|}
\hline Variables & $\begin{array}{c}\text { eGFR } \geq 60 \\
n=695\end{array}$ & $\begin{array}{c}\text { eGFR } 30-59 \\
n=108\end{array}$ & $\begin{array}{c}\text { eGFR }<30 \\
n=28\end{array}$ & $p$ Value \\
\hline One-vessel disease & $340(48.9 \%)$ & $35(32.4 \%)$ & $11(39.3 \%)$ & 0.004 \\
\hline Three-vessel disease & $144(20.7 \%)$ & $31(28.7 \%)$ & $10(35.7 \%)$ & 0.039 \\
\hline LAD & $426(61.3 \%)$ & $75(69.4 \%)$ & $16(57.1 \%)$ & 0.228 \\
\hline Left main & $28(4 \%)$ & $9(8.3 \%)$ & $2(7.1 \%)$ & 0.119 \\
\hline Bifurcation & $28(4 \%)$ & $6(5.6 \%)$ & $1(3.6 \%)$ & 0.752 \\
\hline SYNTAX score (SS) & $15.77( \pm 9.95)$ & $19.27( \pm 10)$ & $21.34( \pm 13.91)$ & $<0.001$ \\
\hline SS $>22$ & $159(22.8 \%)$ & $37(34.3 \%)$ & $11(39.3 \%)$ & 0.007 \\
\hline SS $>32$ & $53(7.6 \%)$ & $9(8.3 \%)$ & $7(25 \%)$ & 0.005 \\
\hline Stent implantation & $655(94.2 \%)$ & $105(97.2 \%)$ & $28(100 \%)$ & 0.195 \\
\hline Total stent lenghts (mm) & $29.99( \pm 20.35)$ & $32.86( \pm 21.29)$ & $34.39( \pm 19.51)$ & 0.238 \\
\hline Maximum Stent Diameter per lesion (mm) & $2.91( \pm 0.82)$ & $2.95( \pm 0.65)$ & $3.18( \pm 0.39)$ & 0,199 \\
\hline Thrombectomy & $102(14.7 \%)$ & $10(9.3 \%)$ & $4(14.3 \%)$ & 0.319 \\
\hline Irradiation time (min) & $11.42( \pm 8.34)$ & $12.36( \pm 10.98)$ & $12.38( \pm 7)$ & 0.511 \\
\hline Contrast medium (mL) & $207.7( \pm 88.23)$ & $190.25( \pm 78.64)$ & $155.04( \pm 70.79)$ & 0.002 \\
\hline Residual SYNTAX score (rSS) & $3.69( \pm 5.73)$ & $6.67( \pm 7.36)$ & $8.64( \pm 10.01)$ & $<0.001$ \\
\hline $\mathrm{rSS}>8$ & $113(16.3 \%)$ & $36(33.3 \%)$ & $13(46.4 \%)$ & $<0.001$ \\
\hline SYNTAX index revascularization (\%) & $80.34( \pm 26.32)$ & $68.9( \pm 28.52)$ & $68.99( \pm 28.31)$ & $<0.001$ \\
\hline SRI $<70 \%$ & $198(28.4 \%)$ & $54(50 \%)$ & $14(50 \%)$ & $<0.001$ \\
\hline Staged PCI & $173(24.9 \%)$ & $31(29 \%)$ & $6(21.4 \%)$ & 0.593 \\
\hline
\end{tabular}

Data are presented as mean \pm SD or $\mathrm{n}(\%)$. eGFR is expressed in $\mathrm{mL} / \mathrm{min} / 1.73 \mathrm{~m}^{2}$. eGFR: estimated Glomerular Filtration rate, LAD: Left anterior descending artery coronary, PCI: Percutaneous coronary intervention, rSS: residual SYNTAX score, SRI: SYNTAX index revascularization, SS: SYNTAX score.

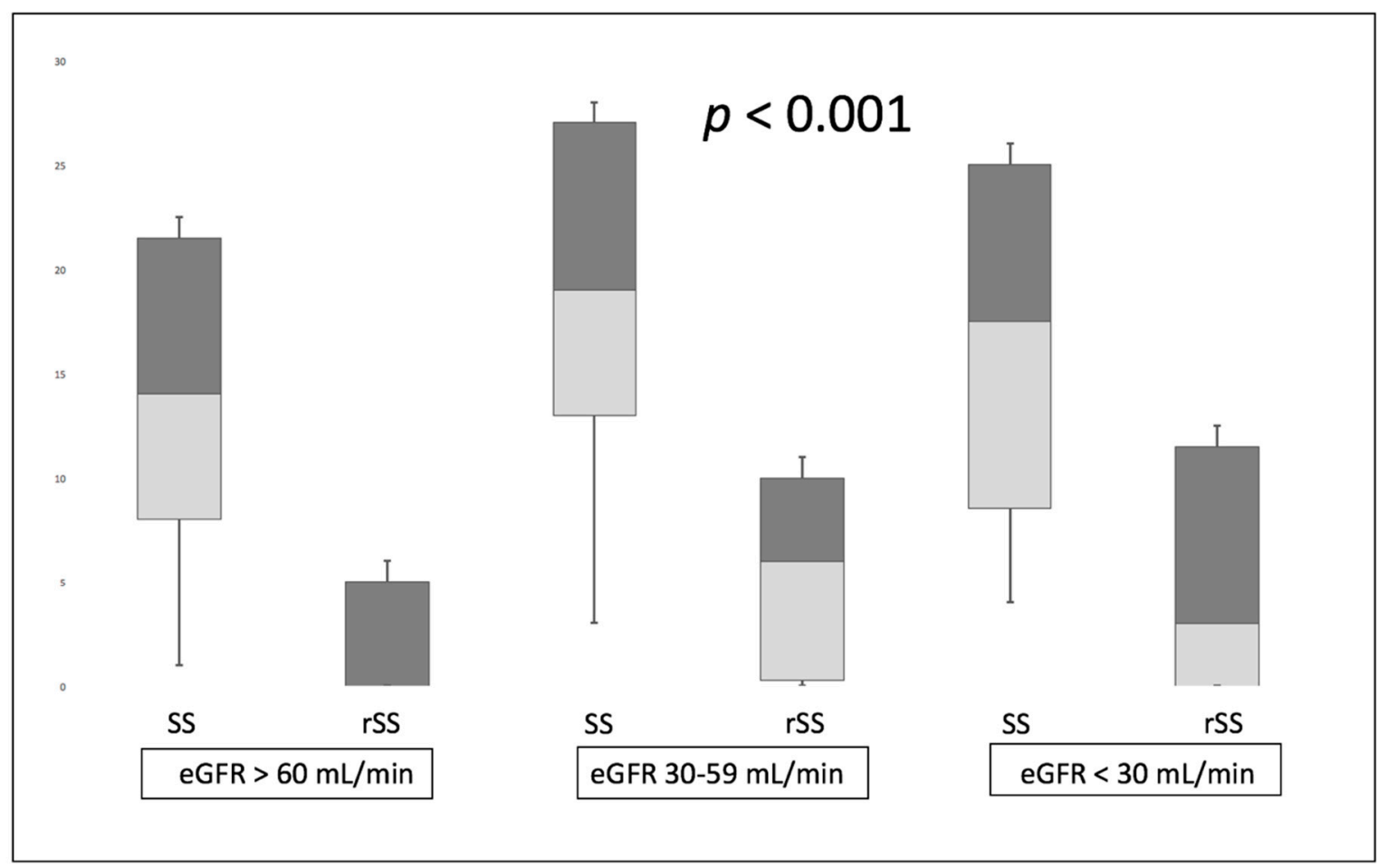

Figure 2. SYNTAX score and rSS according to renal function. SS: SYNTAX score; rSS: residual SYNTAX score. 


\subsection{Impact of CKD on Cardiovascular Outcomes}

Clinical outcomes were available in 826 patients (99.39\%) with a mean follow-up of 324 days. Five patients were lost to follow-up. Major adverse cardiac events (MACE) at 1 year occurred in 121 patients $(14.5 \%)$.

The rates of all-cause death, cardiovascular mortality, rehospitalization for heart failure and MACE were significantly higher among patients with CKD compared to those with preserved eGFR (Figure 3, Table 4).

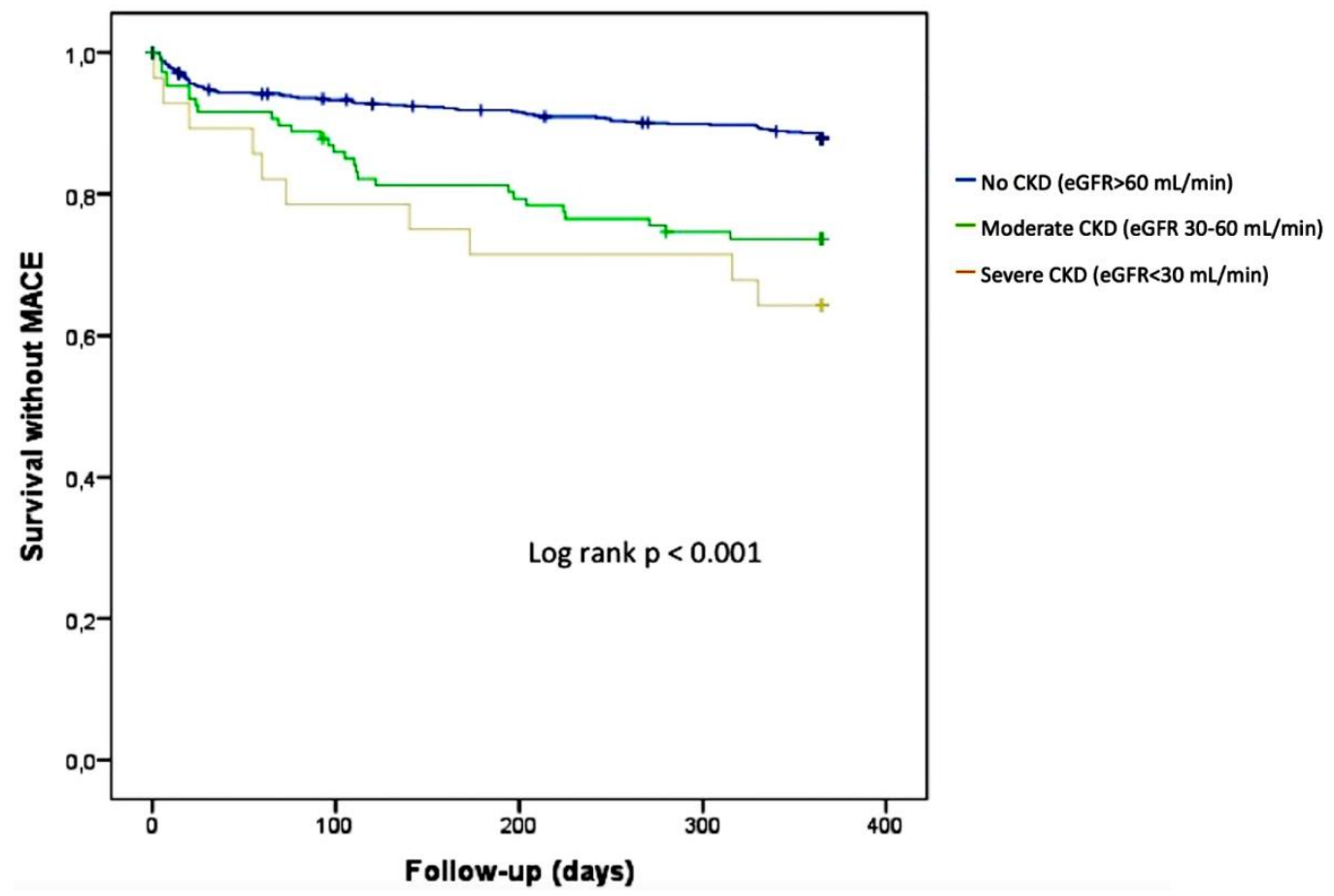

Figure 3. Kaplan Meier analysis for the probability of MACE event-free survival according to renal function.

Table 4. Outcomes at one-year follow-up according to baseline renal function.

\begin{tabular}{|c|c|c|c|c|}
\hline Events & $\begin{array}{c}\text { eGFR } \geq 60 \\
n=695\end{array}$ & $\begin{array}{c}\text { eGFR } 30-59 \\
n=108\end{array}$ & $\begin{array}{c}\text { eGFR }<30 \\
n=28\end{array}$ & $p$ Value \\
\hline MACE & $83(12 \%)$ & $28(25.9 \%)$ & $10(35.7 \%)$ & $<0.001$ \\
\hline All cause mortality & $25(3.6 \%)$ & $12(11.1 \%)$ & $7(25 \%)$ & $<0.001$ \\
\hline Cardiac death & $11(1.6 \%)$ & $7(6.5 \%)$ & $3(10.7 \%)$ & $<0.001$ \\
\hline STEMI & $7(1 \%)$ & $1(0.9 \%)$ & $0(0 \%)$ & 0.866 \\
\hline NSTEMI & $33(4.8 \%)$ & $8(7.4 \%)$ & $2(7.1 \%)$ & 0.457 \\
\hline Stroke & $9(1.3 \%)$ & $6(4.6 \%)$ & $0(0 \%)$ & 0.036 \\
\hline Stent thrombosis & $12(1.7 \%)$ & $1(0.9 \%)$ & $0(0 \%)$ & 0.657 \\
\hline Hemorrhagic events & $56(8.3 \%)$ & $13(12 \%)$ & $5(17.9 \%)$ & 0.096 \\
\hline Heart Failure & $22(3.2 \%)$ & $17(15.7 \%)$ & $6(21.4 \%)$ & $<0.001$ \\
\hline Angina & $52(7.5 \%)$ & $9(8.3 \%)$ & $3(10.7 \%)$ & 0.792 \\
\hline
\end{tabular}

Data are expressed as $\mathrm{n}(\%)$. eGFR is expressed in $\mathrm{mL} / \mathrm{min} / 1.73 \mathrm{~m}^{2}$. eGFR: estimated Glomerular Filtration rate, MACE: Major adverse cardiac events, NSTEMI: Non ST-segment elevation myocardial infarction, STEMI: ST-segment elevation myocardial infarction.

\subsection{Impact of Incomplete Revascularization ( $r S S>8$ ) on Cardiovascular Outcomes}

Compared to the patients with optimal revascularization, patients with $\mathrm{rSS}>8$ had significantly higher risk of all cause and cardiovascular mortality, STEMI, CABG, stroke, angina, heart failure and MACE (Figure 4, Table 5). 


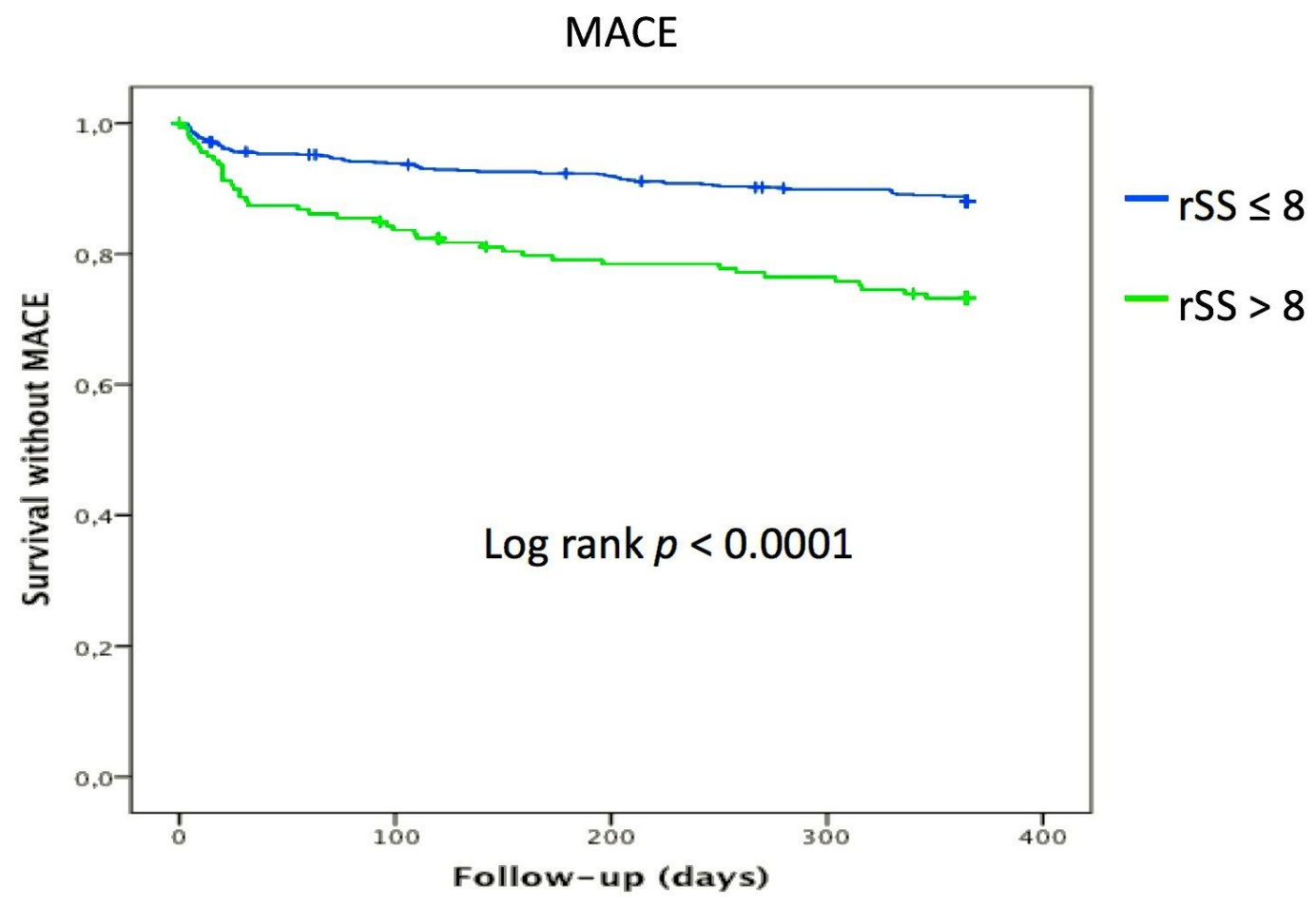

Figure 4. Kaplan Meier analysis for the probability of MACE event-free survival according to rSS.

Table 5. Outcomes at one year follow-up according to rSS.

\begin{tabular}{cccc}
\hline Events & $\begin{array}{c}\text { rSS } \leq \mathbf{8} \\
\mathbf{n} \mathbf{6 6 9}(\mathbf{8 0 . 5} \%)\end{array}$ & $\begin{array}{c}\text { rSS }>\mathbf{8} \\
\boldsymbol{n} \mathbf{\mathbf { 1 6 2 }} \mathbf{( 1 9 . 5 \% )}\end{array}$ & $p$ Value \\
\hline MACE & $79(11.8 \%)$ & $42(25.9 \%)$ & $<0.001$ \\
All cause mortality & $23(3.4 \%)$ & $21(13 \%)$ & $<0.001$ \\
Cardiac death & $8(1.2 \%)$ & $13(8 \%)$ & $<0.001$ \\
STEMI & $4(0.6 \%)$ & $4(2.5 \%)$ & 0.029 \\
NSTEMI & $35(5.2 \%)$ & $8(5 \%)$ & 0.893 \\
CABG & $3(0.4 \%)$ & $5(3.1 \%)$ & 0.002 \\
Stroke & $6(0.9 \%)$ & $9(5.6 \%)$ & $<0.001$ \\
Stent thrombosis & $11(1.6 \%)$ & $2(1.2 \%)$ & 0.703 \\
Angina & $41(6.1 \%)$ & $23(14.2 \%)$ & 0.001 \\
Heart Failure & $24(3.6 \%)$ & $21(13 \%)$ & $<0.001$ \\
Hemorrhagic events & $55(8.2 \%)$ & $19(11.7 \%)$ & 0.160 \\
\hline
\end{tabular}

eGFR is expressed in $\mathrm{mL} / \mathrm{min} / 1.73 \mathrm{~m}^{2}$. Data are expressed as $n$ (\%). eGFR: estimated Glomerular Filtration rate, CABG: Cardiac artery bypass grafting, MACE: Major adverse cardiac events, NSTEMI: Non ST-segment elevation myocardial infarction, STEMI: ST-segment elevation myocardial infarction.

\subsection{Predictors of $r S S>8$ (Incomplete Revascularization)}

By univariate Cox regression analysis, age, diabetes mellitus, hypertension, dyslipemia, atrial fibrillation, peripheral artery disease, prior NSTEMI, CKD, three-vessel disease were significant predictors of a rSS $>8$ (Table 6). Multivariate Cox regression analysis identified age $>75$ years, diabetes mellitus, CKD and three-vessel disease as independent predictors of a rSS $>8$ (Table 6). 
Table 6. Univariate and multivariate analysis of risk factors associated with rSS $>8$.

\begin{tabular}{ccccc}
\hline & \multicolumn{2}{c}{ Univariate } & \multicolumn{2}{c}{ Multivariate } \\
\hline Variables & HR (95\% CI) & $p$ Value & HR (95\% CI) & $P$ Value \\
\hline Age & $1.04(1.03-1.06)$ & $<0.001$ & $1.03(1.01-1.04)$ & $<0.001$ \\
STEMI & $0.95(0.67-1.34)$ & 0.765 & & \\
NSTEMI & $0.82(0.73-1.48)$ & 0.819 & & \\
Diabetes mellitus & $2.14(1.48-3.11)$ & $<0.001$ & $1.50(0.97-2.30)$ & 0.064 \\
Hypertension & $2.09(1.43-3.05)$ & $<0.001$ & $1.05(0.66-1.66)$ & 0.823 \\
Dyslipidemia & $1.58(1.12-2.25)$ & 0.009 & $0.92(0.61-1.14)$ & 0.732 \\
Current Smoking & $0.63(0.44-0.91)$ & 0.014 & & \\
Obesity & $0.93(0.63-1.38)$ & 0.732 & & \\
Atrial Fibrillation & $2.01(1.15-3.53)$ & 0.014 & $0.99(0.52-1.92)$ & 0.978 \\
PAD & $2.01(1.14-3.49)$ & 0.015 & $1.00(0.52-1.92)$ & 0.983 \\
Prior STEMI & $1.62(0.98-2.67)$ & 0.062 & & \\
Prior NSTEMI & $2.35(1.37-4.02)$ & 0.002 & $1.31(0.70-2.45)$ & 0.383 \\
Previous angioplasty & $1.21(0.77-1.89)$ & 0.41 & & \\
CKD (eGFR $<$ 60 mL/min $\left./ \mathrm{m}^{2}\right)$ & $3.10(2.07-4.67)$ & $<0.001$ & $1.75(1.07-2.86)$ & 0.024 \\
Three-vessel disease & $5.05(3.49-7.33)$ & $<0.001$ & $4.29(2.89-6.37)$ & $<0.001$ \\
\hline
\end{tabular}

eGFR is expressed in $\mathrm{mL} / \mathrm{min} / 1.73 \mathrm{~m}^{2}$. HR: Hazard ratio, CI: Confidence interval, CKD: Chronic kidney disease, eGFR: estimated Glomerular Filtration rate, PAD: peripheral artery disease, NSTEMI: Non ST-segment elevation myocardial infarction, STEMI: ST-segment elevation myocardial infarction.

\subsection{Predictors of Cardiac Death and MACE}

By univariate Cox analysis, Killip class $\geq \mathrm{II}, \mathrm{CKD}$, atrial fibrillation, peripheral artery disease, $\mathrm{LVEF} \leq 40 \%$, three-vessel disease, $\mathrm{SS}>22, \mathrm{rSS}>8$ and SRI $<70 \%$ were significant predictors of cardiac death at one year follow-up (Table 7). The occurrence of MACE was directly related to NSTEMI, hypertension, diabetes mellitus, $\mathrm{CKD}$, atrial fibrillation, peripheral artery disease, Killip class $\geq \mathrm{II}$, LVEF $\leq 40 \%$, three-vessel disease, SS $>22$ and rSS $>8$. By contrast, STEMI, the use of ACE/ARB, beta blocker or statin and one-vessel disease were associated with a lower risk of MACE (Table 7). Multivariate Cox regression analysis identified a rSS $>8$, but not diabetes mellitus or CKD, as an independent predictor of cardiac death and MACE (Table 8).

Table 7. Univariate and multivariate analysis of risk factors associated with cardiac death.

\begin{tabular}{ccccc}
\hline & \multicolumn{2}{c}{ Univariate } & \multicolumn{2}{c}{ Multivariate } \\
\hline Events & HR (95\% CI) & $p$ Value & HR (95\% CI) & $p$ Value \\
\hline Age & $1.09(1.05-1.14)$ & $<0.001$ & $1.04(0.99-1.09)$ & 0.064 \\
Gender (female) & $1.27(0.49-3.27)$ & 0.620 & & \\
STEMI & $1.07(0.45-2.56)$ & 0.866 & & \\
NSTEMI & $1.16(0.49-2.76)$ & 0.733 & & \\
Diabete mellitus & $1.29(0.49-3.31)$ & 0.602 & & \\
Hypertension & $2.21(0.81-6.02)$ & 0.123 & & \\
Dyslipidemia & $1.45(0.61-3.45)$ & 0.390 & & \\
Current Smoking & $0.26(0.07-0.89)$ & 0.031 & & \\
Family history of CAD & $0.64(0.19-2.17)$ & 0.474 & & 0.326 \\
Obesity & $0.91(0.33-2.45)$ & 0.844 & & \\
Atrial Fibrillation & $5.39(2.07-14.03)$ & 0.001 & $1.69(0.59-4.82)$ & \\
PAD & $4.72(1.83-12.18)$ & 0.001 & $1.84(0.63-5.42)$ & \\
CKD (eGFR $<60$ mL/min) & $4.21(1.77-9.99)$ & 0.001 & $0.77(0.28-2.15)$ & \\
SBP & $0.99(0.97-1.01)$ & 0.566 & & 0.226 \\
DBP & $1.02(0.98-1.05)$ & 0.380 & & \\
Killip $\geq 2$ & $7.02(2.91-16.95)$ & $<0.001$ & $1.92(0.67-5.55)$ & \\
LVEF $\leq 40 \%$ & $5.65(2.38-13.41)$ & $<0.001$ & $1.60(0.57-4.52)$ & \\
Acute Kidney Injury & $7.81(3.31-18.39)$ & $<0.001$ & $2.57(0.94-6.97)$ & \\
Aspirin & $0.004(0.002-0.01)$ & $<0.001$ & & \\
Colpidogrel & $0.87(0.36-2.11)$ & 0.762 & & \\
ACE/ARB & $0.08(0.034-0.191)$ & $<0.001$ & & \\
Betablocker & $0.08(0.03-0.181)$ & $<0.001$ & & \\
Statin & $0.03(0.01-0.08)$ & $<0.001$ & & \\
& & & & \\
\hline & & & \\
\end{tabular}


Table 7. Cont.

\begin{tabular}{ccccc}
\hline & \multicolumn{2}{c}{ Univariate } & \multicolumn{2}{c}{ Multivariate } \\
\hline Events & HR (95\% CI) & $p$ Value & HR (95\% CI) & $p$ Value \\
\hline One-vessel disease & $0.26(0.08-0.76)$ & 0.014 & & \\
Three-vessel disease & $2.86(1.20-6.78)$ & 0.017 & & \\
Left main & $1.06(0.14-7.89)$ & 0.956 & & \\
LAD & $2.69(0.91-7.98)$ & 0.075 & & \\
SYNTAX score $>$ 22 & $4.29(1.81-10.17)$ & 0.001 & & \\
SYNTAX score $>32$ & $6.39(2.58-15.86)$ & $<0.001$ & & \\
SRI $<70 \%$ & $3.55(1.47-8.56)$ & 0.005 & 3.014 \\
rSS $>8$ & $7.22(2.99-17.44)$ & $<0.001$ & $3.28-8.93)$ & 0.014 \\
\hline
\end{tabular}

eGFR is expressed in $\mathrm{mL} / \mathrm{min} / 1.73 \mathrm{~m}^{2}$. CAD: Coronary artery disease, CI: Confidence interval, DBP: Diastolic blood pressure, eGFR: estimated Glomerular Filtration rate, HR: Hazard ratio, LAD: Left anterior descending artery coronary, LVEF: Left ventricle ejection fraction, PAD: Peripheral artery disease, NSTEMI: Non ST-segment elevation myocardial infarction, rSS: Residual SYNTAX score, SBP: Systolic blood pressure, SRI: SYNTAX index revascularization, STEMI: ST-segment elevation myocardial infarction.

Table 8. Univariate and multivariate analysis of risk factors associated with MACE.

\begin{tabular}{|c|c|c|c|c|}
\hline \multirow[b]{2}{*}{ Variables } & \multicolumn{2}{|c|}{ Univariate } & \multicolumn{2}{|c|}{ Multivariate } \\
\hline & HR $(95 \%$ CI) & $p$ Value & HR $(95 \%$ CI) & $p$ Value \\
\hline Age & $1.03(1.01-1.04)$ & $<0.001$ & $1.01(0.99-1.02)$ & 0.669 \\
\hline Gender (female) & $1.19(0.80-1.78)$ & 0.386 & & \\
\hline STEMI & $0.59(0.41-0.85)$ & 0.005 & & \\
\hline NSTEMI & $1.84(1.29-2.63)$ & 0.001 & $1.74(1.20-2.53)$ & 0.003 \\
\hline Diabete mellitus & $1.68(1.15-2.44)$ & 0.007 & $1.10(0.73-1.67)$ & 0.645 \\
\hline Hypertension & $1.96(1.31-2.94)$ & 0.001 & $1.33(0.85-2.09)$ & 0.401 \\
\hline Dyslipidemia & $1.37(0.95-1.96)$ & 0.087 & & \\
\hline Current Smoking & $0.74(0.50-1.08)$ & 0.116 & & \\
\hline Family history of CAD & $0.99(0.64-1.54)$ & 0.976 & & \\
\hline Obesity & $0.95(0.63-1.43)$ & 0.817 & & \\
\hline Prior NTSEMI & $2.23(1.37-3.64)$ & 0.001 & & \\
\hline Prior STEMI & $1.75(1.08-2.83)$ & 0.022 & & \\
\hline Prior unstable angina & $1.84(0.96-3.51)$ & 0.031 & & \\
\hline Prior Stroke & $1.92(1.08-3.41)$ & 0.026 & & \\
\hline Atrial Fibrillation & $2.15(1.28-3.59)$ & 0.003 & $1.17(0.68-2.04)$ & 0.574 \\
\hline PAD & $2.13(1.28-3.51)$ & 0.003 & $1.11(0.64-1.92)$ & 0.710 \\
\hline CKD $(\mathrm{eGFR}<60 \mathrm{~mL} / \mathrm{min})$ & $2.36(1.59-3.49)$ & $<0.001$ & $1.26(0.78-2.02)$ & 0.340 \\
\hline Killip $\geq 2$ & $2.39(1.65-3.48)$ & $<0.001$ & $1.24(0.78-1.98)$ & 0.358 \\
\hline $\mathrm{LVEF} \leq 40 \%$ & $2.42(1.67-3.51)$ & $<0.001$ & $1.74(1.11-2.71)$ & 0.015 \\
\hline Acute Kidney Injury & $2.44(1.61-3.70)$ & $<0.001$ & $1.46(0.91-2.35)$ & 0.115 \\
\hline Aspirin & $0.03(0.01-0.05)$ & $<0.001$ & & \\
\hline Colpidogrel & $1.52(1.06-2.17)$ & 0.021 & & \\
\hline $\mathrm{ACE} / \mathrm{ARB}$ & $0.55(0.38-0.82)$ & 0.003 & & \\
\hline Betablocker & $0.38(0.24-0.62)$ & $<0.001$ & & \\
\hline Statin & $0.19(0.12-0.32)$ & $<0.001$ & & \\
\hline One-vessel disease & $0.49(0.33-0.71)$ & $<0.001$ & & \\
\hline Three-vessel disease & $2.38(1.64-3.44)$ & $<0.001$ & & \\
\hline Left main & $2.66(1.49-4.73)$ & 0.001 & & \\
\hline LAD & $1.95(1.29-2.95)$ & 0.002 & & \\
\hline SYNTAX score $>22$ & $2.18(1.51-3.13)$ & $<0.001$ & & \\
\hline Syntax score $>32$ & $2.67(1.65-4.32)$ & $<0.001$ & & \\
\hline SRI $<70 \%$ & $1.46(1.01-2.11)$ & 0.040 & & \\
\hline $\mathrm{rSS}>8$ & $2.46(1.69-3.58)$ & $<0.001$ & $1.63(1.08-2.46)$ & 0.02 \\
\hline
\end{tabular}

eGFR is expressed in $\mathrm{mL} / \mathrm{min} / 1.73 \mathrm{~m}^{2}$. ACE: Angiotensin converting-enzyme inhibitor, ARB: Angiotensin II receptor blockers, CAD: Coronary artery disease, CI: Confidence interval, CKD: Chronic kidney disease, eGFR: estimated Glomerular Filtration rate, HR: Hazard ratio, LAD: Left anterior descending artery coronary, LVEF: Left ventricle ejection fraction, PAD: peripheral artery disease, NSTEMI: Non ST-segment elevation myocardial infarction, rSS: residual SYNTAX score, SRI: SYNTAX index revascularization, STEMI: ST-segment elevation myocardial infarction. 


\section{Discussion}

The current report drawn from a cohort of 831 consecutive ACS patients, is the first study to quantify the extent and severity of coronary artery disease prior and after staged revascularization by PCI according to the extent of kidney disease. The main finding of this study is that CKD is predictive of incomplete revascularization during ACS as assessed by higher rSS values. The importance of rSS on adverse outcomes is emphasized by the evidence that incomplete revascularization, but not CKD or diabetes mellitus, is a strong independent predictor of one-year mortality, cardiac mortality and MACE following PCI.

\subsection{Impact of CKD on Adverse Cardiovascular Outcomes Following PCI}

Patients with CKD represent an increasing proportion of the population undergoing PCI and up to $40 \%$ of the patients treated for ACS [11]. Several studies and registries in the context of ACS and elective PCI have reported a negative association between CKD and mortality, stent thrombosis, post procedural ischemic events on the one hand, and bleeding events on the other hand [11-15]. The mechanisms through which CKD affects the clinical outcome are believed to be multifactorial and may include increased cardiovascular comorbidities such as diabetes mellitus, enhanced oxidative stress, endothelial dysfunction, persistent micro-inflammation, coronary calcification, higher burden of yellow atherosclerotic plaques and platelet activation $[16,17]$. Other factors contributing to adverse outcomes include insufficient use of well-proven therapies, lower inhibition by thienopyridines $[7,18]$ and reluctance to perform coronary angiography, which is mostly motivated by advanced age, the presence of multiple comorbidities, bleeding risk and an enhanced risk of contrast induced nephropathy as eGFR declines. A first insight into the deleterious role of undergoing revascularization in CKD came from Chertow and coworkers. They demonstrated that inappropriately low rates of coronary angiography in elderly individuals with renal insufficiency indicated by itself, an enhanced risk of adverse outcomes [19]. Likewise, another study has suggested in patients with multivessel CAD that complete revascularization by drug-eluting stents (DES) reduced cardiac death and myocardial infarction incidence. The observed benefit of complete revascularization was maximal in diabetes, low ejection fraction and low eGFR patients [20]. Challenging this view, a recent retrospective multivariate analysis has shown that incomplete revascularization was not independently predictive of adverse outcomes in octogenarians and supports a more conservative approach [21]. In the present study, increased baseline coronary artery disease burden was evidenced as kidney function declined and witnessed by higher prevalence of three-vessel disease, baseline SS, \% of patients with SS $>22$ or 32 (Table 3). Baseline SS calculated in the whole cohort $(16.4 \pm 10)$ was higher than the one proposed in the original report by Genereux $(12.8 \pm 6)$ and derived from a post hoc analysis of the ACUITY (Acute Catheterization and Urgent Intervention Triage Strategy) trial [4]. In our work, staged PCI was equally performed among the 3 groups but could not totally blunt baseline differences, rSS, and $\mathrm{SRI}<70 \%$, which happened to be at their highest levels in patients with severe CKD. The proportion of patients with rSS $>8$ observed in this real-world study $(19.5 \%)$ is in line with the original report by Genereux (18.7\%) [4] and a recent report by Khan in STEMI patients (21.8\%) [1]. The question whether CKD, or incomplete revascularization associated with CKD independently hamper cardiovascular outcomes has not been addressed up to now. In our study, CKD along with history of diabetes mellitus, age $>75$ years and three-vessel disease were demonstrated to be independent predictors of rSS $>8$. In an attempt to delineate the respective contribution of CKD, diabetes mellitus and incomplete revascularization on cardiac mortality and MACE following ACS, multivariate analysis was performed. The present data suggest that incomplete revascularization plays a major role in the determination of adverse outcomes; and both CKD and diabetes mellitus are no longer independently associated with cardiac mortality/MACE when rSS was taken into account. The most commonly accepted reasons for incomplete revascularization in CKD patients are generally multifactorial and may include the following: (i) ignorance of NSTE-ACS risk (despite paradoxically high calculated GRACE-risk scores), (ii) ignorance of potential treatment benefit in these patients, (iii) fear of an 
immediate complication such as contrast induced nephropathy or bleeding, (iv) co-morbidities, and (v) absence of population-specific clinical trial data.

\subsection{Impact of Incomplete Revascularization on Adverse Cardiovascular Outcomes Following PCI}

The present study extends previous reports that have demonstrated a strong relationship between incomplete revascularization and worse cardiovascular outcomes in several circumstances including ACS $[1,4,22,23]$. However, a marked variability in the prognostic value of incomplete revascularization has been reported and possibly reflects the lack of consensus on the definition of optimal revascularization [20]. Other important drawbacks of these studies, including the present one, rely on the definition of incomplete revascularization based on percent diameter stenosis derived from visual estimation. As nicely depicted by the Fractional Flow Reserve Versus Angiography for Multivessel Disease Evaluation (FAME) study, nearly one third of angiographically significant stenosis were actually hemodynamically not significant [24]. Therefore, the poor correlation between the angiogram and the presence of vessel related ischemia could cloud the relationship between the degree of revascularization and clinical outcome [25]. In line with this view, recent data derived from the FAME study have demonstrated that residual angiographic lesions that are not functionally significant $($ FFR $>0.80)$ do not reflect residual ischemia or predict adverse outcomes [26]. Despite these limitations, the validation of incomplete revascularization in clinical practice assessed by a rSS $>8$ was originally addressed by Genereux and coworkers as an important marker of adverse outcomes in ACS patients.

The rSS aims to represent a reliable measurement of the myocardial ischemia burden. It depends on the location of the coronary disease, the importance in supplying blood to the jeopardized myocardium, and the anatomic complexity associated with the obstructive disease [23].

In the work by Genereux, age, insulin-treated diabetes, hypertension, smoking, elevated biomarkers or ST-segment deviation and lower ejection fraction were more frequent in patients with incomplete revascularization [4]. Although the demonstration that incomplete revascularization is associated with a poorer prognosis does not necessarily imply that revascularization of residual stenosis visible on the angiogram improves prognosis, these data might indicate that the tracking of residual ischemia is highly warranted in high risk patients such as those with CKD. In that setting, given the high prevalence of incomplete revascularization observed in CKD patients (33.3\% and $46.4 \%$ ) following staged PCI, alternative strategies of revascularization such as CABG or hybrid PCI plus CABG should be evaluated by the heart team unless the interventional cardiologist is confident that the level of incomplete revascularization will be low. Indeed, a recent large meta-analysis has emphasized that complete revascularization is more commonly achieved with CABG [27].

\subsection{Study Limitations}

This study includes several limitations. (i) As the SYNTAX score is not designed to assess angiographic complexity in patients with $C A B G$, those patients were excluded from our analysis. (ii) The SYNTAX score was assessed by only one investigator (A.K.). Intra-individual variability assessed by a new evaluation of 10 random angiograms was less than $5 \%$. (iii) The small number of patients with severe CKD limited the interpretation of our data. (iv) The functional assessment of angiographic stenosis by FFR was not systematically investigated. (v) The SYNTAX score is basically aimed to assess the technical complexity of percutaneous coronary interventions. It is therefore conceivable that some of the features used to calculate the score have a marked impact on the success of the procedure (e.g., tortuosity of calcification) but may not be added as prognostic information for future atherosclerosis-related events. (vi) The cardiovascular events were not adjudicated by an independent committee. Finally, as with similar evaluations of registry data, there are inherent limitations for this type of study, mainly related to known or unknown confounding factors. The results of this report should therefore be considered as hypothesis generating and prospective trials are required to further evaluate the importance of achieving complete revascularization in CKD patients. 


\section{Conclusions}

In acute coronary syndrome, CKD is predictive of incomplete revascularization following staged PCI procedures. The importance of rSS on adverse outcomes is emphasized by our demonstration that incomplete revascularization, but not CKD nor diabetes mellitus, is a strong independent predictor of one-year mortality, cardiac mortality and MACE following PCI.

Author Contributions: Conceptualization, T.C. and O.M.; Methodology, T.C. and O.M.; Validation, L.J., P.O. and O.M.; Formal Analysis, J.R., V.A.H., S.H., M.K., L.J., P.O. and O.M.; Investigation, T.C., A.K. and O.M.; Data Curation, T.C., A.K., A.T., B.M., J.R., V.A.H., S.H. and O.M.; Writing-Original Draft Preparation, T.C., A.K. and O.M.; Writing-Review \& Editing, A.T., B.M. and O.M.; Supervision, O.M.

Conflicts of Interest: The authors declare no conflict in interest.

\section{Abbreviations}

ACS, acute coronary syndrome; BNP, B-type natriuretic peptide; CABG, coronary artery bypass grafting; CAD, coronary arterial disease; CKD, chronic kidney disease; CRP, C-reactive protein; DES, drug-eluting stent(s); eGFR, estimated glomerular filtration rate; LVEF, left ventricle ejection fraction; MACE, major adverse cardiac events; MVD, multi vessel disease; NSTEMI, non-STEMI; PCI, percutaneous coronary intervention; rSS, residual SYNTAX score; SRI, Syntax revascularization index; SS, SYNTAX score; STEMI, ST segment elevation myocardial infarction; SYNTAX, Synergy Between PCI With Taxus and Cardiac Surgery.

\section{References}

1. Khan, R.; Al-Hawwas, M.; Hatem, R.; Azzalini, L.; Fortier, A.; Joliecoeur, E.M.; Tanguay, J.F.; Lavoie-L'allier, P.; Ly, H.Q. Prognostic impact of the residual SYNTAX score on in-hospital outcomes in patients undergoing primary percutaneous coronary intervention. Catheter. Cardiovasc. Interv. 2016, 88, 740-747. [CrossRef] [PubMed]

2. Hoebers, L.P.; Vis, M.M.; Claessen, B.E.; Van der Schaaf, R.J.; Kikkert, W.J.; Baan, J., Jr.; de Winter, R.J.; Piek, J.J.; Tijssen, J.G.; Dangas, G.D.; et al. The impact of multivessel disease with and without a co-existing chronic total occlusion on short- and long-term mortality in ST-elevation myocardial infarction patients with and without cardiogenic shock. Eur. J. Heart Fail. 2013, 15, 425-432. [CrossRef] [PubMed]

3. Van der Schaaf, R.J.; Claessen, B.E.; Vis, M.M.; Hoebers, L.P.; Koch, K.T.; Baan, J., Jr.; Meuwissen, M.; Engstrom, A.E.; Kikkert, W.J.; Tijjsen, J.G.; et al. Effect of multivessel coronary disease with or without concurrent chronic total occlusion on one-year mortality in patients treated with primary percutaneous coronary intervention for cardiogenic shock. Am. J. Cardiol. 2010, 105, 955-959. [CrossRef] [PubMed]

4. Genereux, P.; Palmerini, T.; Caixeta, A.; Rosner, G.; Green, P.; Dressler, O.; Xu, K.; Parise, H.; Mehran, R.; Serruys, P.W.; et al. Quantification and impact of untreated coronary artery disease after percutaneous coronary intervention: The residual SYNTAX (Synergy Between PCI with Taxus and Cardiac Surgery) score. J. Am. Coll. Cardiol. 2012, 59, 2165-2174. [CrossRef] [PubMed]

5. Loutfi, M.; Ayad, S.; Sobhy, M. Impact of the Residual SYNTAX Score on Outcomes of Revascularization in Patients with ST-Segment Elevation Myocardial Infarction and Multivessel Disease. Clin. Med. Insights Cardiol. 2016, 10, 29-35. [CrossRef] [PubMed]

6. Levey, A.S.; Bosch, J.P.; Lewis, J.B.; Greene, T.; Rogers, N.; Roth, D. Modification of Diet in Renal Disease Study Group. A more accurate method to estimate glomerular filtration rate from serum creatinine: A new prediction equation. Ann. Intern. Med. 1999, 130, 461-470. [CrossRef] [PubMed]

7. Morel, O.; El Ghannudi, S.; Jesel, L.; Radulescu, B.; Meyer, N.; Wiesel, M.L.; Caillard, S.; Campia, U.; Moulin, B.; Gachet, C.; et al. Cardiovascular mortality in chronic kidney disease patients undergoing percutaneous coronary intervention is mainly related to impaired P2Y12 inhibition by clopidogrel. J. Am. Coll. Cardiol. 2011, 57, 399-408. [CrossRef]

8. El Ghannudi, S.; Ohlmann, P.; Meyer, N.; Wiesel, M.L.; Radulescu, B.; Chauvin, M.; Bareiss, P.; Gachet, C.; Morel, O. Impact of P2Y12 inhibition by clopidogrel on cardiovascular mortality in unselected patients treated by percutaneous coronary angioplasty: A prospective registry. JACC Cardiovasc. Interv. 2010, 3, 648-656. [CrossRef] 
9. Coskun, U.; Orta Kilickesmez, K.; Abaci, O.; Kocas, C.; Bostan, C.; Yildiz, A.; Baskurt, M.; Arat, A.; Ersanli, M.; Gurmen, T. The relationship between chronic kidney disease and SYNTAX score. Angiology 2011, 62, 504-508. [CrossRef]

10. Yan, L.Q.; Guo, L.J.; Zhang, F.C.; Gao, W. The relationship between kidney function and angiographically-derived SYNTAX score. Can. J. Cardiol. 2011, 27, 768-772. [CrossRef]

11. Tsai, T.T.; Messenger, J.C.; Brennan, J.M.; Patel, U.D.; Dai, D.; Piana, R.N.; Anstrom, K.J.; Eisenstein, E.L.; Dokholyan, R.S.; Peterson, E.D.; et al. Safety and efficacy of drug-eluting stents in older patients with chronic kidney disease: A report from the linked CathPCI Registry-CMS claims database. J. Am. Coll. Cardiol. 2011, 58, 1859-1869. [CrossRef] [PubMed]

12. Latif, F.; Kleiman, N.S.; Cohen, D.J.; Pencina, M.J.; Yen, C.H.; Cutlip, D.E.; Moliterno, D.J.; Nassif, D.; Lopez, J.J.; Saucedo, J.F. In-hospital and 1-year outcomes among percutaneous coronary intervention patients with chronic kidney disease in the era of drug-eluting stents: A report from the EVENT (Evaluation of Drug Eluting Stents and Ischemic Events) registry. JACC Cardiovasc. Interv. 2009, 2, 37-45. [CrossRef] [PubMed]

13. Machecourt, J.; Danchin, N.; Lablanche, J.M.; Fauvel, J.M.; Bonnet, J.L.; Marliere, S.; Foote, A.; Quesada, J.L.; Eltchaninoff, H.; Vanzetto, G. Risk factors for stent thrombosis after implantation of sirolimus-eluting stents in diabetic and nondiabetic patients: The EVASTENT Matched-Cohort Registry. J. Am. Coll. Cardiol. 2007, 50, 501-508. [CrossRef] [PubMed]

14. Akashi, N.; Sakakura, K.; Watanabe, Y.; Noguchi, M.; Taniguchi, Y.; Yamamoto, K.; Wada, H.; Momomura, S.I.; Fujita, H. The comparison of clinical outcomes in patients with acute myocardial infarction and advanced chronic kidney disease on chronic hemodialysis versus off hemodialysis. Heart Vessel. 2018, 33, 713-721. [CrossRef] [PubMed]

15. Nakahashi, H.; Kosuge, M.; Sakamaki, K.; Kiyokuni, M.; Ebina, T.; Hibi, K.; Tsukahara, K.; Iwahashi, N.; Kuji, S.; Oba, M.S.; et al. Combined impact of chronic kidney disease and contrast-induced nephropathy on long-term outcomes in patients with ST-segment elevation acute myocardial infarction who undergo primary percutaneous coronary intervention. Heart Vessel. 2017, 32, 22-29. [CrossRef] [PubMed]

16. Shroff, G.R.; Herzog, C.A. Coronary Revascularization in Patients with CKD Stage 5D: Pragmatic Considerations. J. Am. Soc. Nephrol. 2016, 27, 3521-3529. [CrossRef] [PubMed]

17. Morel, O.; Muller, C.; Jesel, L.; Moulin, B.; Hannedouche, T. Impaired platelet P2Y12 inhibition by thienopyridines in chronic kidney disease: Mechanisms, clinical relevance and pharmacological options. Nephrol. Dial. Transplant. 2013, 28, 1994-2002. [CrossRef]

18. Muller, C.; Caillard, S.; Jesel, L.; El Ghannudi, S.; Ohlmann, P.; Sauleau, E.; Hannedouche, T.; Gachet, C.; Moulin, B.; Morel, O. Association of estimated GFR with platelet inhibition in patients treated with clopidogrel. Am. J. Kidney Dis. Off. J. Natl. Kidney Found. 2012, 59, 777-785. [CrossRef]

19. Chertow, G.M.; Normand, S.L.; McNeil, B.J. “Renalism”: Inappropriately low rates of coronary angiography in elderly individuals with renal insufficiency. J. Am. Soc. Nephrol. 2004, 15, 2462-2468. [CrossRef]

20. Chung, J.W.; Park, K.H.; Lee, M.H.; Park, K.W.; Park, J.S.; Kang, H.J.; Koo, B.K.; Kwon, Y.W.; Kim, H.S. Benefit of complete revascularization in patients with multivessel coronary disease in the drug-eluting stent era. Circ. J. 2012, 76, 1624-1630. [CrossRef]

21. Yazji, K.; Abdul, F.; Elangovan, S.; Ossei-Gerning, N.; Choudhury, A.; Cockburn, J.; Anderson, R.; Mamas, M.; Kinnaird, T. Comparison of the Effects of Incomplete Revascularization on 12-Month Mortality in Patients $<80$ Compared With $>/=80$ Years Who Underwent Percutaneous Coronary Intervention. Am. J. Cardiol. 2016, 118, 1164-1170. [CrossRef] [PubMed]

22. Rosner, G.F.; Kirtane, A.J.; Genereux, P.; Lansky, A.J.; Cristea, E.; Gersh, B.J.; Weiz, G.; Parise, H.; Fahy, M.; Mehran, R.; et al. Impact of the presence and extent of incomplete angiographic revascularization after percutaneous coronary intervention in acute coronary syndromes: The Acute Catheterization and Urgent Intervention Triage Strategy (ACUITY) trial. Circulation 2012, 125, 2613-2620. [CrossRef] [PubMed]

23. Farooq, V.; Serruys, P.W.; Bourantas, C.V.; Zhang, Y.; Muramatsu, T.; Feldman, T.; Holmes, D.R.; Mack, M.; Morice, M.C.; Stahle, E.; et al. Quantification of incomplete revascularization and its association with five-year mortality in the synergy between percutaneous coronary intervention with taxus and cardiac surgery (SYNTAX) trial validation of the residual SYNTAX score. Circulation 2013, 128, 141-151. [CrossRef] [PubMed] 
24. Tonino, P.A.; De Bruyne, B.; Pijls, N.H.; Siebert, U.; Ikeno, F.; van't Veer, M.; Klauss, V.; Manoharan, G.; Engstrom, T.; Oldroyd, K.G.; et al. Fractional flow reserve versus angiography for guiding percutaneous coronary intervention. N. Engl. J. Med. 2009, 360, 213-224. [CrossRef] [PubMed]

25. De Bruyne, B. Multivessel disease: From reasonably incomplete to functionally complete revascularization. Circulation 2012, 125, 2557-2559. [CrossRef] [PubMed]

26. Kobayashi, Y.; Nam, C.W.; Tonino, P.A.; Kimura, T.; De Bruyne, B.; Pijls, N.H.; Fearon, W.F. The Prognostic Value of Residual Coronary Stenoses After Functionally Complete Revascularization. J. Am. Coll. Cardiol. 2016, 67, 1701-1711. [CrossRef] [PubMed]

27. Garcia, S.; Sandoval, Y.; Roukoz, H.; Adabag, S.; Canoniero, M.; Yannopoulos, D.; Brilakis, E.S. Outcomes after complete versus incomplete revascularization of patients with multivessel coronary artery disease: A meta-analysis of 89,883 patients enrolled in randomized clinical trials and observational studies. J. Am. Coll. Cardiol. 2013, 62, 1421-1431. [CrossRef]

(C) 2019 by the authors. Licensee MDPI, Basel, Switzerland. This article is an open access article distributed under the terms and conditions of the Creative Commons Attribution (CC BY) license (http://creativecommons.org/licenses/by/4.0/). 